Irish Math. Soc. Bulletin

Number 72, Winter 2013, 37-37

ISSN 0791-5578

\title{
CODES OVER RINGS AND APPLICATIONS TO COMBINATORICS
}

\author{
ALISON SNEYD
}

This is an abstract of the $\mathrm{PhD}$ thesis Codes over Rings and Applications to Combinatorics written by Alison Sneyd under the supervision of Dr Eimear Byrne at the School of Mathematical Sciences, University College Dublin and submitted in December 2012.

Generalising a classical result of Delsarte [3], it was recently shown that certain codes over finite Frobenius rings with two nonzero homogeneous weights determine strongly regular graphs [1]. This thesis gives constructions for infinite families of two-weight codes over Frobenius rings that result in strongly regular graphs. Some of the codes constructed do not have prime power order and the strongly regular graphs they yield therefore cannot arise from the classical construction over finite fields. Many of the strongly regular graphs constructed are shown to be isomorphic to graphs resulting from orthogonal arrays. In addition, relationships between the parameters of a two-weight code and the eigenvalues of the corresponding strongly regular graph are developed, allowing existence criteria for two-weight codes to be derived. The results of a computer search for two-weight codes are presented.

\section{REFERENCES}

[1] E. Byrne, M. Greferath and T. Honold: Ring geometries, two-weight codes, and strongly regular graphs. Des. Codes Cryptogr., 48. 2008.

[2] E. Byrne, M. Kiermaier and A. Sneyd: Properties of codes with two homogeneous weights Finite Fields Appl., 18. 2012.

[3] Ph. Delsarte: Weights of linear codes and strongly regular normed spaces. Discrete Math., 3. 1972.

School of Mathematical Sciences, University College Dublin

E-mail address, A. Sneyd: alison.sneyd@ucd.ie

2010 Mathematics Subject Classification. 94B25,94B05,94B60,05E3.

Key words and phrases. Codes over Rings, Strongly Regular Graphs.

Received on 16-12-2013.

Support from SFI is gratefully acknowledged. 